\section{The Community Oncologist: Part of the Solution}

Medical oncology is an incredibly interesting and rewarding field of medicine. In no other area has change been so dramatic over such a short time. Furthermore, in no other area have the fruits of basic scientific labor been applied in such a tangible way to improve the care of patients. As a community oncologist, it has been my pleasure to be on the frontlines, as a witness to and participant in this reformation. However, we do have our challenges. Community oncologists are being assaulted on several fronts, frequently in the context of the uncontrolled costs of cancer care. Community oncologists are often cast in the role of villain, as part of the problem rather than part of the solution.

In my role as a Medical Director for US Oncology, I have been involved in discussions and debates surrounding many of these contentious issues, from reimbursement reform to Centers for Medicare \& Medicaid (CMS) and FDA policy to comparative effectiveness research. I have been frustrated by my perception that community oncologists are being marginalized in these discussions, despite the expectation that these same community providers will enthusiastically support, contribute to, and ultimately implement these programs.

At a recent meeting about what comparative effectiveness will actually look like, I asked in exasperation, "As a provider, what do you want me to do?"

I offer here a modest proposal for what we can and should do.

\section{Commitment to Evidence-Based Medicine}

The rapid rise in the costs associated with health care is certainly mind-boggling. With the growth rate outstripping the rise in gross domestic product, few feel this is sustainable. Cancer care has been a convenient scapegoat in this discussion. This is undoubtedly related to the monstrous sticker prices that have been attached to many of the new treatment approaches. Examples like Provenge, Avastin, and Erbitux fan the flames. And perhaps what have been most damning are the marginal returns on investment and the perceived lack of value. But cancer treatment spending represents only $5 \%$ to $10 \%$ of the health care dollar, and chemotherapy only about $25 \%$ of cancer care spending. Most cancer spending is related to hospital-related costs, and much is associated with futile treatment at the end of life.

The issue, then, is not whether an individual treatment is too expensive. We may legitimately spend thousands on a bone marrow transplant. Rather, the issue is evidence-based use of available therapies. We can no longer recommend a therapy based on a theory of potential benefit. This new approach will take courage and true grit in the current era of entitlement and consumerism. Community oncologists will need the support of academic oncologists, not only through authorship of clinical guidelines and education of providers and payors, but also through consistency in second opinions and institutional practices.

At US Oncology, we have been aggressively developing and implementing a network-wide evidence-based pathways program that is linked to our electronic medical record, allowing for both decision support and eventually outcomes measurement. I believe the entire oncology community must be committed to evidence-based medicine. We must be viewed as responsible stewards of our precious resources, our patients, and the health care funds to take care of them. We also need to become more forward thinking and aggressive in the application of advance care planning and end of life care. For many adults with advanced cancer, cure is not an achievable goal, and at some point palliation with chemotherapy isn't either. "Hospice" shouldn't have the

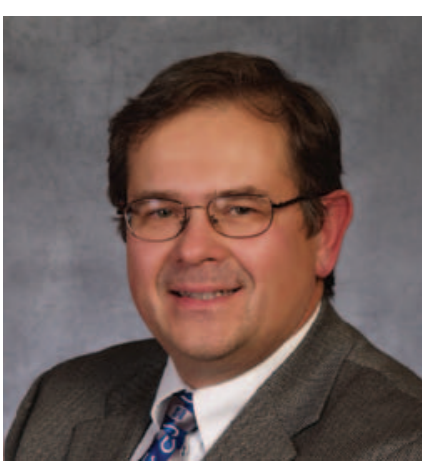

Michael A. Kolodziej, MD

Michael A. Kolodziej, MD, is a physician with New York Hematology Oncology in Albany, New York. Dr. Kolodziej attended college and medical school at Washington University in St. Louis. He completed internal medicine and hematology-oncology training at the University of Pennsylvania in Philadelphia. After completing training, Dr. Kolodziej joined the faculty at the University of Oklahoma School of Medicine where he was an associate professor. He joined New York Oncology in 1998. He currently serves as Chief of Oncology at St. Peter's Hospital, a 400-bed community hospital in Albany. He has been an active member of the US Oncology Pharmacy and Therapeutics committee, on the executive committee for the past 9 years, and chairman for the past 5 years. He has served as Medical Director for Oncology Services for US Oncology for the past 4 years. In this role, he has helped direct the implementation of the USON clinical pathways initiative, the integration of the USON EMR into this program, and the development of the USON disease management and advanced care planning programs, which is now known as Innovent Oncology. He maintains an active practice, with special emphasis on breast cancer and prostate cancer.

The ideas and viewpoints expressed in this commentary are those of the author and do not necessarily represent any policy, position, or program of the NCCN. 
emotional baggage it currently does. Community oncologists are looking to partner with academic oncologists as leaders in this area. We are not, after all, death panelists.

\section{Defining What Constitutes Value}

A discussion of cost cannot be complete without consideration of current reimbursement models. Most community oncologists in office-based practice administer chemotherapy via a buy-and-bill model, in which the practice derives financial benefit (actually survival) from the margin on chemotherapy. This is believed to create a perverse incentive in which more chemotherapy means more money, and ipso facto inappropriate prescribing behavior. Recent publications on the use of androgen-ablative therapy are used as evidence for this phenomenon. But these publications are horribly flawed by absence of rich clinical data that can be married to the claims data. Or, as I learned a long time ago, correlation does not prove causality.

Most community oncologists would embrace a reformed reimbursement methodology that rewards cognitive skills, quality performance, and outcomes. However, reaching that objective from where we are now is no simple matter. Quality measures are difficult to come by, and the ones we currently have are process measures, not outcomes measures. Some providers are counting on the proposed Accountable Care Organizations to get us there. But remember that most community oncology groups have fewer than 5 providers. The only hope for these small groups is to join a hospital. There are no integrated delivery systems where I work. And even if hospitals were the answer (and I do not think they are), we are at the "pilot" stage. Nobody knows what these are really going to look like or if they will work. To be truthful, oncology providers are still working to define what constitutes value.

\section{A System in Need of Repair}

Clinical research and the upcoming comparative effectiveness research need a happy, healthy marriage between community and academic oncologists to reach full potential. Recently, the Institute of Medicine published an honest and critical assessment of everything wrong with clinical research in oncology. Most community oncologists have known for many years that the system is broken, and most have little confidence that the proposed changes in clinical research will significantly resolve the challenges that hinder clinical research in the practice setting. There is sad blindness to the Willie Sutton bank robbery wisdom about where the money (or in this case, patient) is. After all, $80 \%$ of all cancer care is administered in the community. Clinical research must be relevant and userfriendly, must reward the participant, and cannot erect reimbursement hurdles.

Comparative effectiveness is even more abstract to community oncologists, and they are not alone in their confusion. Of the top 100 topics identified by the Institute of Medicine for comparative effectiveness research, only a handful are oncology-related, and the ability of medical oncologists to contribute to these is questionable. How are we to contribute? Do we need to enter data into the electronic health record, which can be queried? Do we contribute to registries? Do we identify patients who are candidates for biomarker research? Who has the time? What is the reward? What do you want me to do?

\section{A United Effort}

Community and academic oncologists are certainly more alike than different. We trained together and share common goals of providing the best possible care for our patients. Although we are not always particularly collegial, curiously, our combined 
The Community Oncologist

ire at the FDA and CMS has brought us together. The REMS explosion has been equally daunting to both parties. Label decisions by the FDA, including the recent Avastin/breast cancer debate, and coverage policies espoused by CMS, including the precedent-setting National Coverage Determination on Provenge, have touched all practicing oncologists. NCCN's success in developing an evidence-based drug compendium, derived from their clinical guidelines and recognized as authoritative by both CMS and several commercial payors, including UnitedHealthcare, has been a major step forward in administrative ease for the community oncologist.

The solution may involve recognition of the fertile common ground. Community oncologists, by and large, are a very pragmatic group. Treating solid tumors in adults forces us to be realists. We respect what our academic colleagues bring to the table, and we embrace the concept of a partnership to help solve some of the challenges in oncology. But in response to the rhetorical question, "What do you want me to do?" don't just tell us what to do. Ask us. 